\title{
Gender difference in early initiation of methamphetamine use among current methamphetamine users in Muse, Northern Shan State, Myanmar
}

Yu Mon Saw ${ }^{1,2^{*}}$, Thu Nandar Saw ${ }^{3,4}$, Junko Yasuoka ${ }^{4}$, Nyein Chan ${ }^{5}$, Nang Pann Ei Kham ${ }^{6}$, Wint Khine ${ }^{7}$, Su Myat Cho ${ }^{1}$ and Masamine Jimba ${ }^{4}$

\begin{abstract}
Background: Globally, methamphetamine (MA) use is a significant public health concern due to unprecedented health effects of its use. However, gender similarities and differences in early age of MA initiation and its risk factors among current MA users have been understudied in a developing country setting.

Methods: A community-based, cross-sectional study was conducted using a computer assisted self-interviewing program from January to March 2013 in Muse, Northern Shan State, Myanmar. A total of 1362 (775 male and 587 female) self-reported current MA users aged between 18 and 35 years were recruited using respondent-driven sampling. Two gender-stratified multiple logistic regression models (models I and II) were done for analysis.

Results: For similarities, $73.0 \%$ of males and $60.5 \%$ of females initiated MA before their 18th birthday. The early age of MA initiation was positively associated with the reasons and places of the first time MA use among both genders. For differences, males [hazard ratio 1.35; 95\% confidence interval, 1.18-1.54] had a significantly higher risk than females to initiate MA at earlier age. Among male users, participants who had bisexual/homosexual preferences were more likely to initiate MA use earlier. In contrast, female users who exchanged sex for money and/or drugs were more likely to initiate MA in earlier age.

Conclusions: More than $60.0 \%$ of male and female participants initiated MA use early; however, males initiated use earlier than females. Although similarities were found among both genders, differences found in key risk factors for early age MA initiation suggest that gender-specific, MA prevention programs are urgently needed in Myanmar.
\end{abstract}

Keywords: Initiation, Methamphetamine, Gender, Drug user, Myanmar

\section{Background}

Amphetamine-type stimulants (ATS) are getting popular among young population, due to their affordable and wide availability $[1,2]$. In 2012, ATS was ranked as the second most commonly used drug worldwide which may be due to its powerful stimulant effects. It acts in the central nervous system: increasing alertness; providing a sense of increased energy, concentration, physical

\footnotetext{
* Correspondence: sawyumon@med.nagoya-u.ac.jp

'Department of Healthcare Administration, Graduate School of Medicine, Nagoya University, 65 Tsurumai-cho, Showa-ku, Nagoya 466-8550, Japan ${ }^{2}$ Nagoya University Asian Satellite Campuses Institute, Nagoya, Japan

Full list of author information is available at the end of the article
}

strength; and creating euphoria [3]. Among different types of ATS, methamphetamine (MA) pills are most commonly used all over the world. Approximately 80 million people used ATS, and 21 million of them were based in East and Southeast Asia [1, 4, 5].

The response to MA is not always the same between males and females. Gender-specific similarities and variations have been reported in many aspects of MA use and health services [6]. For example, both males and females may use MA for sexual enhancement [7], but females are more likely to use MA pills for weight loss $[8,9]$. Females also tend to show more dependence and commitment to MA, whereas males are more likely to 
use other drugs if MA is not available [10, 11]. Females seek access to MA abuse treatment more often than males, [2] and tend to be more open and responsive to this treatment [12]. Moreover, being compared to male users, female users seemed to have a tendency to initiate MA, cocaine, and amphetamine at earlier ages, and to report medical problems caused by drug abuse than male users $[11,13]$.

Initiation of MA occurs sometime during adolescence. Initiation at such an early age is often associated with a variety of negative outcomes in adulthood including drug addiction, criminal, and violent behavior, and healthrelated problems [14, 15]. In addition, individuals who start using drug in their adolescence may continue using it through their adulthood. Even when they stop, they might revert into using it later in adulthood $[16,17]$. Thus delaying the initiation of MA use, in addition to preventing MA use, could be important in controlling the adverse effects of MA use.

In Myanmar, the government has tried to control opiate drug use, but has shown less attention to ATS. This has led to Myanmar becoming a major ATS manufacturing country in Southeast Asia; in particular, it has produced MA pills known as "Yama or Yaba" [1, 4]. These drugs are mainly produced for the international market, but MA is also used within the country. In 2009, MA pills were seized in 16 out of 17 administrative regions in Myanmar, highlighting their nationwide availability [18]. Although, opium and heroin remain the most widely used drugs in Myanmar [18].

Despite increasing MA use, little has been studied about the initiation of MA, particularly the gender similarities and differences in it, in Myanmar. Delaying the initiation is critical in this country, but the risk factors for initiation have been adequately studied. It is important to know the age at which MA use is initiated and factors associated with this in Myanmar. Such information could help establish effective strategy for controlling MA use in the country. Moreover, evidence is particularly lacking in relation to gender-specific factors associated with the age of initiation among drug users in Myanmar. Therefore, this study was conducted to elucidate the gender-specific factors associated with the early initiation of MA use.

\section{Methods}

\section{Study settings and participants}

The study area was Muse city in Northern Shan State, Myanmar. The city is located close to the Chinese border, neighboring Ruili city in the Chinese province of Yunnan, and accommodates a highly mobile population of migrant workers. Northern Shan State has a long history of opium and heroin use [19], and MA use has also become a growing problem in this area over the past decade. The majority of MA pill seizures in Myanmar are routinely made near to the production areas in the border towns of Northern Shan State.

A community-based, cross-sectional survey was carried out from January to March 2013. In total, 1385 MA users (782 males and 603 females) were recruited. Of them, 23 participants ( 7 males and 16 females) were excluded from final data analysis due to withdrawal from the study or who missed to answer the outcome question. A final sample of 1362 MA users (775 males; 587 females) were considered for analysis. The study inclusion criteria were: (1) self-reported MA users aged between 18 and 35 years, (2) those who had used methamphetamine drugs in the last 3 months, (3) those who had no withdrawal symptoms and were not under the influence of drugs at the time of interview, (4) those who are able to read the Myanmar language, and (5) those who voluntarily participated in the study by giving an informed consent.

A computer assisted self-interviewing (CASI) method was applied, in which participants read and respond to survey questions on a computer screen. Since CASI is a self-administered computer program, it improves data collection concerning socially undesirable behaviors [20-22]. Each CASI section took 35-45 $\mathrm{min}$ to complete. Various private settings were used for data collection, based on the participants' preference, such as in a motel room, at the participant's house, at their place of drug use, at their place of work, or in the project's car. For each interview, two or more research team members visited the interview place and set up computer for CASI.

\section{Data collection procedure and recruitment protocol}

The study participants were recruited by respondentdriven sampling (RDS) [23]. First, an MA user was recruited from a local drug user network as the first study participant from the first initial recruitment arm. In total, eight initial recruitment arms were recruited with the help of the National Drug User Network, local men who had sex with men (MSM) network, brothels, local highway car associations, and local youth volunteer groups. The three initial recruitment arms stopped either at second or third wave out of eight initial recruitment arms (4 males and 4 females) [24]. In total, 4095 coupons were distributed and 1427 coupons were returned. Among returned coupons, 62 coupons that presented after two-week coupon expiration date were excluded from the study.

The same recruitment protocol was used to recruit male and female MA users, and they turned to recruit both genders. They also recruited MA users from different settings of the population including MSM, FSWs, students, laborers, housewives, and highway drivers. The 
already recruited MA users recruited three other MA user friends after completing their interviews. Each respondent was allowed to recruit up to three other people to participate in the survey within the two-week coupon expiration date.

All the participants received 2000 Kyats (approx. US\$ 2.5) as an incentive directly following their interviews. They were also eligible to receive a secondary incentive: a steel cup (for the students, laborers, housewives, and highway drivers) or lubricant gel (for FSWs and MSM) that was equivalent to 900 Kyats (approx. US\$ 1.2) if their recruited participants completed their interviews [24]. However, MA users also allowed to choose a steel cup or lubricant gel depending on their preference as a secondary incentive. The other respondents were given the same opportunities as the first respondents for further recruitment and incentives.

\section{Measures}

The questionnaire was adapted from ones created by the United Nations Office on Drugs and Crime [25]. It was translated from English to the Myanmar language and was pre-tested among a sample of MA users in October 2012. Back-translation was done from Myanmar language to English before and after the pre-test to ensure semantic equivalence. A questionnaire was modified based on the results of the pre-test to make it more understandable and easier for participants to answer. Three Myanmar public health experts, who are working on drug use and sexual behavior issues in Myanmar, also reviewed the study questionnaire.

An MA user was defined as such if he/she had used MA at least three times in the 90 days prior to the interview [26]. The outcome variable was the age at which MA use began, which was measured as a binary variable (early versus late), based on the average age of high school completion, which was 17 years old. At this age, most of the students become independent, as they start working or go to college/university [27]. Independent living may lead to poor parental supervision and monitoring which may be responsible for the increased risk of the initiation of substance use $[28,29]$. The early age of initiation for MA use was set up as 17 years or younger; then it was dichotomized as " 1 " and " 0 " otherwise. Other covariates included ethnicity, behaviors related to MA use (i.e., reason for first-time MA use, sources of access to MA during first use, and route of administration during first use), sexual behaviors [having ever exchanged sex for money or drugs, been diagnosed with a sexually transmitted infection (STI), and had multiple sexual partners within the past 6 months], having ever had suicidal thoughts, and instances of previous suicide attempts.

\section{Statistical analysis}

The Statistical Package for the Social Sciences (SPSS) 18.0 software (Chicago, Illinois, USA) was used for statistical analyses. First, a descriptive analysis was run for socio-demographic characteristics, sexual risk behaviors, and characteristics related to MA use by gender. The Kaplan-Meier curve was plotted, and the log-rank test performed to compare gender differences regarding early initiation of MA use. To determine factors associated with early age of MA initiation, two gender-stratified multiple logistic regression models (models I and II) were performed. In all the analyses, the level of significance was set at $p<0.05$ (two-tailed).

\section{Ethical considerations}

The study protocol and consent procedure were approved by the Research Ethics Committee of the Graduate School of Medicine, the University of Tokyo, Tokyo, Japan (Ref. no: 10006/2012), and the Ethical Review Committee, the Defence Services Medical Research Centre, Nay Pyi Taw, Myanmar (Document No: $1 / 2 /$ IRB-7/2013). The purpose of the study and CASI interview procedures were clearly explained to each participant prior to the interview by research team members. For individual interviews, the privacy needs and personal preferences of each respondent were given special attention. All the participants were given time to decide on their voluntary participation and involvement. They were also informed that they could skip answering any question that they did not want to answer and could withdraw from participation at any time during or after the interview without penalty. If participants fully understood and decided to participate, they were requested to read about informed consent on the computer screen and click "agree to participate in this survey" to answer the survey questions. A computer-based informed consent form mentioning the study objectives and purpose of the research was obtained from all respondents, and the confidentiality of the entire data were carefully maintained.

\section{Results}

\section{Socio-demographic characteristics of participants}

The socio-demographic characteristics of all the participants are shown in Table 1. Of 1362 participants, $56.9 \%(n=775)$ were males, and their mean age was 23.4 years [standard deviation (SD) 3.6], and the mean age of females was 22.7 (SD 3.4). More than half of males $(54.3 \%)$ and females $(54.2 \%)$ were aged between 21-25 years. Among 1362, 936 (68.7\%) reported as being single, with the remainder reporting as either currently married $(426,31.3 \%)$ or ever married. Regarding educational status, 607 (44.6\%) 
Table 1 Socio-demographic characteristics of participants $(N=1,362)$

\begin{tabular}{|c|c|c|c|c|c|c|c|}
\hline \multirow[t]{2}{*}{ Characteristics } & \multicolumn{2}{|c|}{ Total $(N=1,362)$} & \multicolumn{2}{|c|}{ Male $(n=775)$} & \multicolumn{2}{|c|}{ Female $(n=587)$} & \multirow[t]{2}{*}{$P$ value } \\
\hline & $n$ & $\%$ & $n$ & $\%$ & $n$ & $\%$ & \\
\hline \multicolumn{8}{|l|}{ Age $^{a}$ (year) } \\
\hline$\leq 20$ & 336 & 24.7 & 165 & 21.3 & 171 & 29.1 & \multirow[t]{4}{*}{$<0.001$} \\
\hline $21-25$ & 739 & 54.3 & 421 & 54.3 & 318 & 54.2 & \\
\hline $26-30$ & 223 & 16.3 & 145 & 18.7 & 78 & 13.3 & \\
\hline$>30$ & 64 & 4.7 & 44 & 5.7 & 20 & 3.4 & \\
\hline \multicolumn{8}{|l|}{ Marital status } \\
\hline Never married & 936 & 68.7 & 553 & 71.4 & 383 & 65.2 & \multirow[t]{2}{*}{0.016} \\
\hline Ever married & 426 & 31.3 & 222 & 28.6 & 204 & 34.8 & \\
\hline \multicolumn{8}{|l|}{ Education } \\
\hline Primary school & 132 & 9.8 & 54 & 7.0 & 80 & 13.6 & \multirow[t]{4}{*}{$<0.001$} \\
\hline Secondary school & 607 & 44.6 & 304 & 39.2 & 303 & 51.6 & \\
\hline High school & 319 & 23.4 & 210 & 27.1 & 109 & 18.6 & \\
\hline University & 302 & 22.2 & 207 & 26.7 & 95 & 16.2 & \\
\hline \multicolumn{8}{|l|}{ Ethnicity } \\
\hline Shan & 461 & 30.6 & 213 & 27.5 & 203 & 34.6 & \multirow[t]{4}{*}{$<0.001$} \\
\hline Kachin & 274 & 20.1 & 158 & 20.4 & 116 & 19.8 & \\
\hline Burma & 409 & 30.0 & 218 & 28.1 & 191 & 32.5 & \\
\hline Others $^{*}$ & 263 & 19.3 & 186 & 24.0 & 77 & 13.1 & \\
\hline \multicolumn{8}{|l|}{ Employment status } \\
\hline Unemployed & 379 & 27.8 & 188 & 24.3 & 191 & 32.5 & \multirow[t]{2}{*}{0.001} \\
\hline Employed & 983 & 72.2 & 587 & 75.7 & 396 & 67.5 & \\
\hline
\end{tabular}

a'Mean age 23.4 years (standard deviation, SD 3.46) for males and 22.7 years (SD 3.38) for females; *(Kyar, Kayin, Chin, Mon, Rakhin, Multi-ethnic, Indians, and the Chinese);

participants reported having a secondary level of education, while $302(22.2 \%)$ reported having university education $(p<0.001)$. In total, $983 \quad(72.2 \%)$ participants were employed at the time of the interview $(p=0.001)$.

\section{Gender differences in age of methamphetamine use}

Figure 1 shows the percentage of ages at initiation of MA use, stratified by gender. A rapid increase in initiation occurred at 14 years for males and 15 years for females. The peak age of initiation was 16 years for

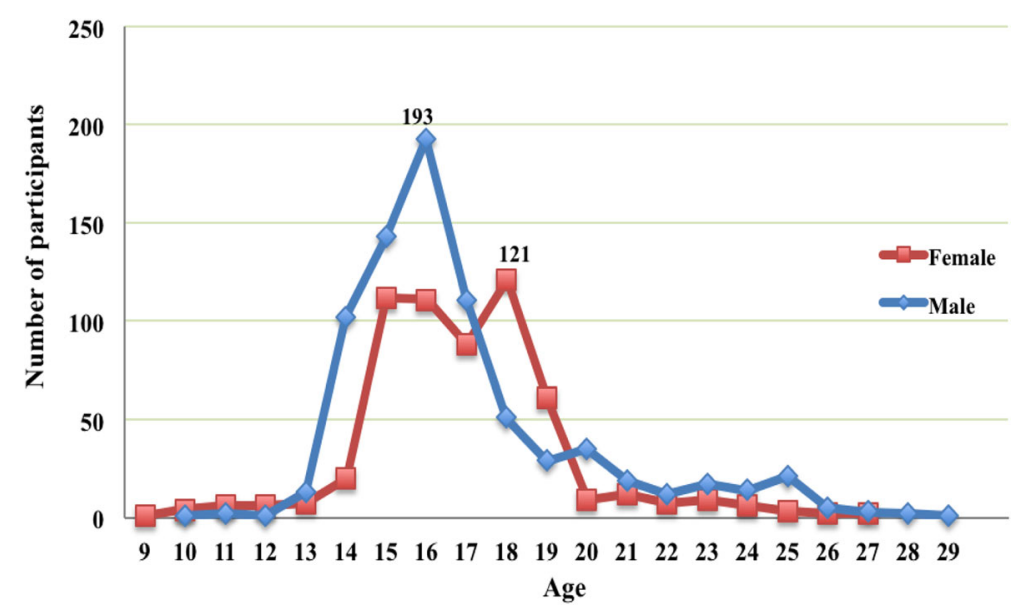

Fig. 1 Age at initiation of methamphetamine use, stratified by gender 
males $(n=193,24.9 \%)$ and 18 years for females $(n=121$, $20.6 \%)$. The median age at initiation of MA use was 16 years (age range: 10-29) for males and 17 years (age range: 9-27) for females.

Figure 2 presents the Kaplan-Meier estimates of age at initiation of MA use by gender. Male users showed a significantly higher risk of early initiation [hazard ratio 1.35; 95\% confidence interval, 1.18-1.54] than did female users.

\section{Multiple logistic regression analyses of early initiation of} methamphetamine use, stratified by gender

Tables 2 and 3 show the results of the multiple logistic regression analyses for males and females. In model I, socio-demographic factors (i.e., age, marital status, education, ethnicity, employment status, and sexual orientation) and characteristics associated with first-time MA use (i.e., place of first MA use, reason for first MA use, source of first-time access to MA, and route of administration during first-time use) were included. In model II, risky sexual behaviors (i.e., having ever exchanged sex for money and/or drugs and having ever been diagnosed with an STI), and suicidal behaviors were added to the variables in model I.

Among males, in model I, factors associated with an increased likelihood of early initiation of MA use included having bisexual/homosexual preferences (adjusted odds ratio $[\mathrm{AOR}]=1.57,95 \% \mathrm{CI}: 1.07-2.29)$, having used MA at entertainment venues ( $\mathrm{AOR}=3.08$; 95\% CI: 2.03-4.65), having used MA either for weight loss or work-related purposes (AOR $=1.61$; 95\% CI: 1.02-2.54), having used MA for the first time because of curiosity about its effects or for fun $(\mathrm{AOR}=2.84$; 95\% CI: 1.74-4.65), and having bought MA by oneself or gotten someone else to buy it $(\mathrm{OR}=2.57 ; 95 \% \mathrm{CI}: 1.77-3.71)$. Conversely, older age $(\mathrm{AOR}=0.39 ; 95 \% \mathrm{CI}: 0.25-0.60)$ and being employed (AOR $=0.72$; 95\% CI: 0.43-1.20) were negatively associated with early initiation of MA use. In model II, the socio-demographic factors and characteristics associated with first-time MA use were statistically significant, and suicide attempts were positively associated with early initiation (AOR = 2.27; 95\% CI: 1.27-4.07) (Table 2).

For females, in model I, multiple logistic regression analysis revealed that participants belonging to the Kachin $(\mathrm{AOR}=0.51 ; 95 \% \mathrm{CI}: 0.31-0.85)$ ethnic groups were less likely to report early initiation of MA use. In contrast, participants who used MA for the first time at an entertainment venue $(\mathrm{AOR}=4.06$; 95\% CI: $2.67-6.17)$ and at a school, dormitory, or the workplace $(\mathrm{AOR}=2.56$; 95\% CI: 1.19-5.53) were more likely to report early initiation of MA use. Similar associations were found among those whose reasons for first-time MA use was either weight loss or work-related purposes $(\mathrm{AOR}=1.89 ; 95 \%$ CI: 1.21-2.93) and curiosity about the drug's effects or for fun $(\mathrm{AOR}=3.30 ; 95 \% \mathrm{CI}: 2.00-5.45)$. In model II, the characteristics associated with first-time MA use were statistically significant and participants who had ever exchanged sex for money and/or drugs $(\mathrm{AOR}=2.04 ; 95 \%$ CI: 1.28-3.26) were more likely to have initiated MA use earlier (Table 3).

\section{Discussion}

In this study, gender similarities and differences were found with regard to both the early initiation of MA use and the associated risk factors. With regard to similarities, a high rate of early initiation was observed in both genders. The places and reasons for first-time MA use

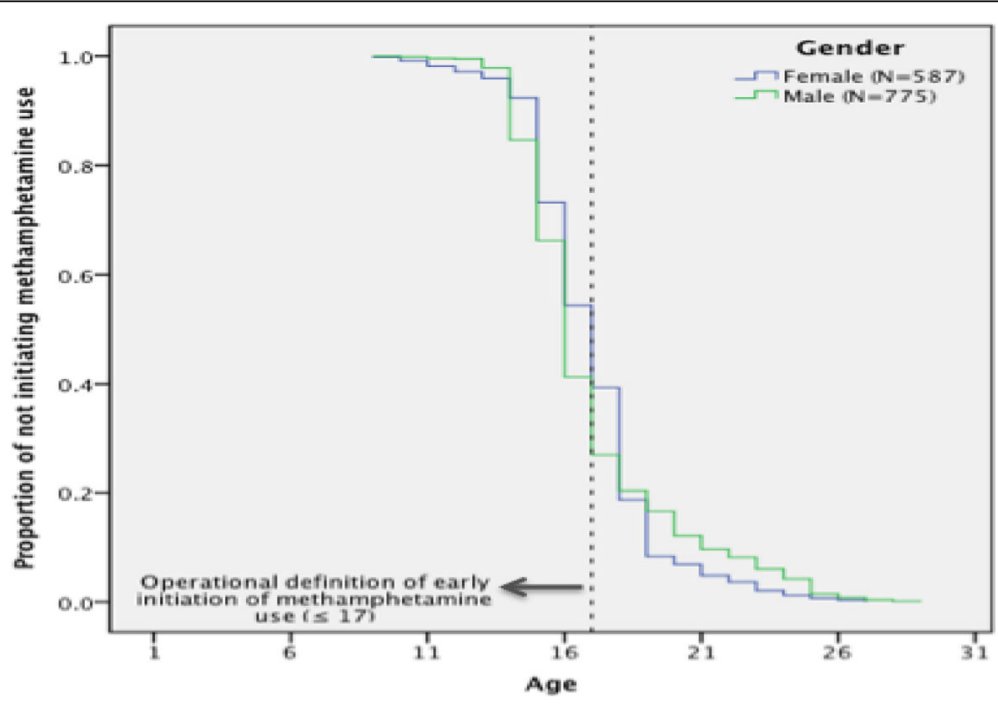

Fig. 2 The Kaplan-Meier curve depicting age at initiation of methamphetamine use by gender 
Table 2 Multiple logistic regression analysis of factors associated with early initiation of methamphetamine use among males $(n=775)$

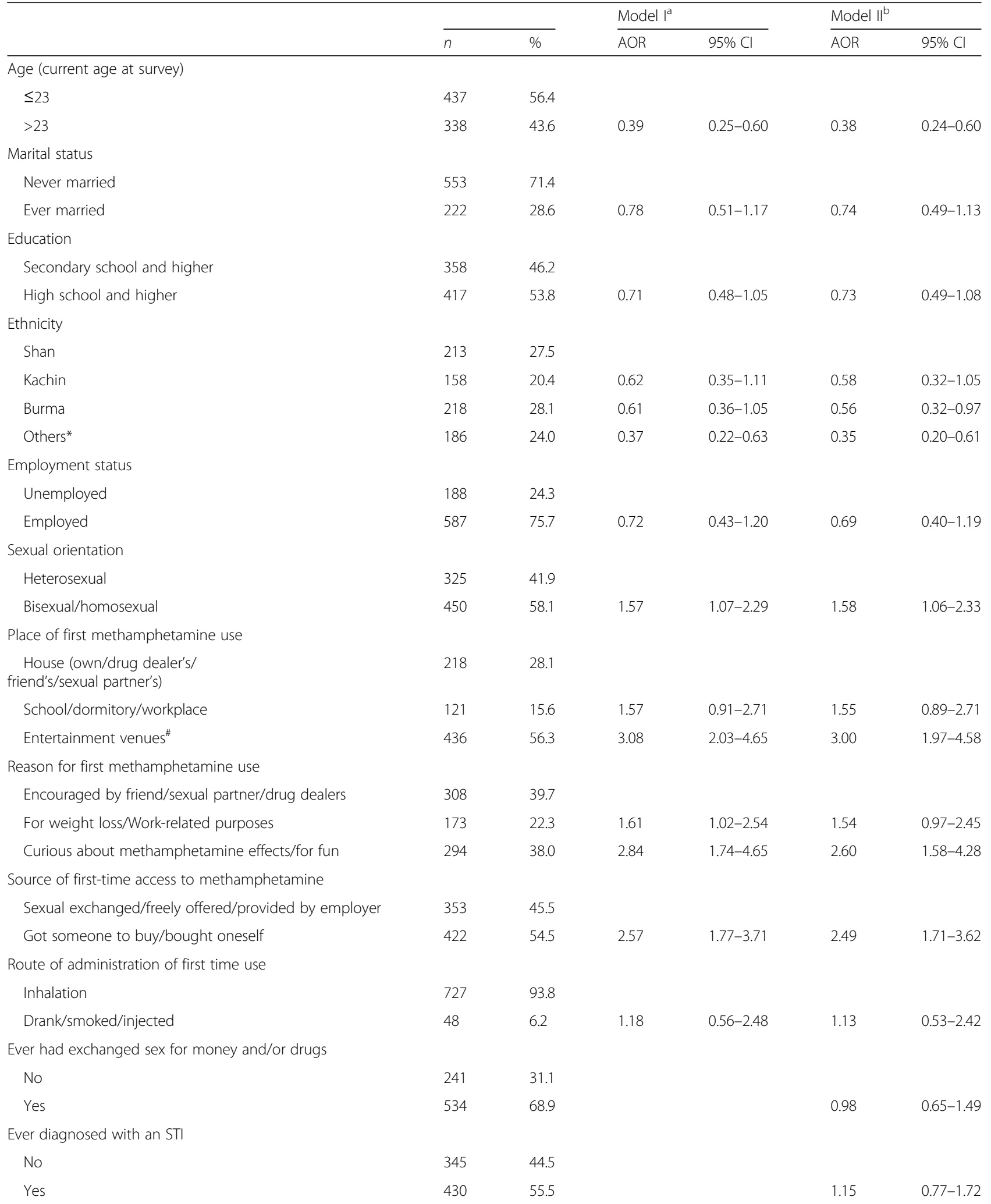


Table 2 Multiple logistic regression analysis of factors associated with early initiation of methamphetamine use among males $(n=775)$ (Continued)

Ever experienced suicidal ideation

No

Yes

Ever attempted suicide

No

Yes
376

399

640

135
48.5

51.5

82.6

17.4

0.82

$0.55-1.22$

OR odd ratio, $\mathrm{Cl}$ confidence interval, $A O R$ adjusted odd ratio; *(Kyar, Kayin, Chin, Mon, Rakhin, Multi-ethnic, Indians, and the Chinese); ${ }^{*}(\mathrm{club} / \mathrm{karaoke} / \mathrm{disco} / \mathrm{restaur}-$ ant/hotel/guest house/game center)

${ }^{a}$ Model I adjusted for age, marital status, education, ethnicity, employment status, sexual orientation, place of first methamphetamine use, reason for first methamphetamine use, source of first methamphetamine access, and route of administration during first-time use

${ }^{\mathrm{b}}$ Model II adjusted for age, marital status, education, ethnicity, employment status, sexual orientation, place of first methamphetamine use, reason for first methamphetamine use, source of first access to methamphetamine, route of administration during first-time use, ever exchanged sex for money and/or drugs,

ever been diagnosed with an STI, ever experienced suicidal ideation, and ever attempted suicide

were positively associated with early initiation of MA use among both males and females. Participants of both genders within the Kachin ethnic group were less likely to initiate MA use at an early age, as compared to those of the Shan ethnic group. With regard to differences, males tended to initiate MA use earlier than females. Among male MA users, those who had bisexual/homosexual preferences had a higher likelihood of reporting early initiation of MA use. Furthermore, male MA users who were employed at the time of the survey were less likely to report early initiation of MA use. Among female MA users, exchanging sex for money and/or drugs were positively associated with early initiation.

One of the marked similarities between the genders was the high percentage of MA use within the sample. More than half of males (73.0\%) and females (60.5\%) initiated MA use before the age of 18 years. Such a high proportion of early initiation of MA use is a major concern, when considering its potential association with a range of health problems later in life [14, 15].

Both males and females belonging to the Kachin ethnic group were less likely to report early initiation of MA use, as compared to participants belonging to the Shan ethnic group. In the United States of America, the age at which use of MA is initiated has also been associated with ethnicity [30]. Similar to MA, early initiation of smoking and the use of alcohol and illicit drugs varied across racial and ethnic groups [31-34].

The place of MA use was also similar across genders. Notably, using MA for the first time at entertainment venues was found to be a potential risk factor for early initiation. This is because entertainment venues, such as bars, clubs, restaurants, motels, Karaoke bars, and game centers provide good opportunities for dealers to sell drugs; drug users can also easily access MA and other illicit drugs at such places $[35,36]$. Furthermore, MA is regarded as a "club or party drug" in the West [36-39]; a similar trend has been observed in Asia. Visiting an entertainment venue is also often associated with drinking and smoking, and the consumption of other illegal substances, such as cocaine and marijuana [40, 41]. In the same way, entertainment venues were found to be one of the important factors in the early use of MA.

Several differences were found between males and females. First, males were more likely to initiate MA use earlier than females were. This is consistent with previous studies conducted in the United States of America $[42,43]$, but not with a study in Taiwan [44] and another study conducted United States of America [45]. Males tend to initiate MA use earlier, as female users are more likely to be stigmatized by society than male users [46]. Drug use is a violation of social norms of behavior; thus, many people consider drug use by females as even worse than by males $[47,48]$. This could also hold true for our study population. In addition, MA use may be influenced by the working environment. Since our study area is close to a Chinese border city, males usually take up jobs during early adolescence, thereby potentially gaining more exposure to MA through their workplaces. One study conducted in the United States of America also indicated that males are more likely than females to come across opportunities to use marijuana, cocaine, hallucinogens, and heroin [49].

Although MA initiation was associated with work purposes for both males and females, differences between males and females in the reasons provided for MA use were worth noticing. For example, females tended to initiate MA use to help them lose body weight, while males initiated it for fun or curiosity about the effects thereof; these results are similar to those of previous studies [11,50-52]. Indeed, MA users sometimes lose weight dramatically when using the drug for a substantial period, as it causes loss of appetite $[42,53,54]$. However, rapid weight gain may occur following the cessation of MA. This makes it more difficult for the affected MA users to stop, resulting in the development of dependence on the drug [55]. 
Table 3 Multiple logistic regression analysis of factors associated with early initiation of methamphetamine use among female participants $(n=587)$

\begin{tabular}{|c|c|c|c|c|c|c|}
\hline & & & Mod & & Mod & \\
\hline & $n$ & $\%$ & $\overline{\mathrm{AOR}}$ & $95 \% \mathrm{Cl}$ & $\mathrm{AOR}$ & $95 \% \mathrm{Cl}$ \\
\hline Age (age during the survey period) & & & & & & \\
\hline$\leq 23$ & 358 & 61.0 & & & & \\
\hline$>23$ & 229 & 39.0 & 0.85 & $0.52-1.39$ & 0.85 & $0.51-1.41$ \\
\hline Marital status & & & & & & \\
\hline Never married & 383 & 65.2 & & & & \\
\hline Ever married & 204 & 34.8 & 0.97 & $0.63-1.48$ & 0.96 & $0.62-1.49$ \\
\hline Education & & & & & & \\
\hline Secondary school and lower & 383 & 65.2 & & & & \\
\hline High school or higher & 204 & 34.8 & 1.40 & $0.93-2.08$ & 1.30 & $0.85-1.99$ \\
\hline Ethnicity & & & & & & \\
\hline Shan & 203 & 34.6 & & & & \\
\hline Kachin & 116 & 19.8 & 0.51 & $0.31-0.85$ & 0.48 & $0.29-0.81$ \\
\hline Burma & 191 & 32.5 & 0.60 & $0.35-1.00$ & 0.61 & $0.36-1.04$ \\
\hline Others* & 77 & 13.1 & 0.63 & $0.34-1.16$ & 0.62 & $0.33-1.16$ \\
\hline Employment status & & & & & & \\
\hline Unemployed & 191 & 32.5 & & & & \\
\hline Employed & 396 & 67.5 & 0.71 & $0.47-1.07$ & 0.65 & $0.42-1.02$ \\
\hline Place of first methamphetamine use & & & & & & \\
\hline House (own/drug dealer's/friend's/sexual partner's) & 373 & 63.5 & & & & \\
\hline School/dormitory/workplace & 39 & 6.7 & 2.56 & $1.19-5.53$ & 2.82 & $1.28-6.19$ \\
\hline Entertainment venues ${ }^{\#}$ & 175 & 29.8 & 4.06 & $2.67-6.17$ & 4.24 & $2.77-6.50$ \\
\hline Reason for first methamphetamine use & & & & & & \\
\hline $\begin{array}{l}\text { Encouraged by friend/sexual } \\
\text { partner/drug dealers }\end{array}$ & 242 & 41.2 & & & & \\
\hline $\begin{array}{l}\text { For weight loss/ } \\
\text { work-related purposes }\end{array}$ & 190 & 32.4 & 1.89 & $1.21-2.93$ & 1.61 & $1.02-2.53$ \\
\hline Curious about methamphetamine effects/for fun & 155 & 26.4 & 3.30 & $2.00-5.45$ & 3.16 & $1.89-5.27$ \\
\hline Source of first-time access to methamphetamine & & & & & & \\
\hline Sexual exchange/freely offered/provided by employer & 354 & 60.3 & & & & \\
\hline Got someone to buy/bought oneself & 233 & 39.7 & 1.09 & $0.74-1.59$ & 1.05 & $0.72-1.56$ \\
\hline Route of administration of first time use & & & & & & \\
\hline Inhalation & 580 & 98.8 & & & & \\
\hline Drank/smoked/injected & 7 & 1.2 & & & & \\
\hline Ever had exchanged sex for money and/or drugs & & & & & & \\
\hline No & 211 & 35.9 & & & & \\
\hline Yes & 376 & 64.1 & & & 2.04 & $1.28-3.26$ \\
\hline Ever diagnosed with an STI & & & & & & \\
\hline No & 308 & 52.5 & & & & \\
\hline Yes & 279 & 47.5 & & & 1.21 & $0.76-1.92$ \\
\hline Ever experienced suicidal ideation & & & & & & \\
\hline No & 243 & 41.4 & & & & \\
\hline Yes & 344 & 58.6 & & & 1.10 & $0.72-1.68$ \\
\hline Ever attempted suicide & & & & & & \\
\hline No & 450 & 76.7 & & & & \\
\hline Yes & 137 & 23.3 & & & 0.98 & $0.60-1.60$ \\
\hline
\end{tabular}

OR odd ratio, Cl confidence interval, AOR adjusted odd ratio; *(Kyar, Kayin, Chin, Mon, Rakhin, Multi-ethnic, Indians, and the Chinese); ${ }^{*}(\mathrm{club} /$ karaoke/restaurant/hotel/guest house/game center)

aModel I adjusted for age, marital status, education, ethnicity, employment status, place of first methamphetamine use, reason for first methamphetamine use, and source of first-time access to methamphetamine

${ }^{\mathrm{b}}$ Model II adjusted for age, marital status, education, ethnicity, employment status, place of first methamphetamine use, reason for first methamphetamine use, source of firsttime access to methamphetamine, ever exchanged sex for money and/or drugs, ever diagnosed with an STI, ever experienced suicidal ideation, and ever attempted suicide 
Gender differences were also found for behaviors posing an HIV risk to the participants. For example, among female MA users, exchanging sex for money and/or drugs was associated with early initiation of MA use. The exchange of sex for money and/or drugs may be a means for their survival. This is supported by the fact that other factors that are associated with initiation of drug use are homelessness or running away from home $[56,57]$. It can also be a means to quell MA addiction [56, 57]. Associations between MA initiation and risky sexual behaviors have an important implication for the fight against HIV/AIDS and other STIs. Thus, it is important to understand the factors associated with high-risk sexual behaviors.

This study provides several important findings and insights. However, some limitations should be noted. First, there is a possibility of recall bias in the reported ages of first MA use, although people may remember this kind of moment better than more ordinary occasions [58]. Second, the possibility of underreporting exists, particularly for sensitive topics such as drug use and sexual behaviors. However, the use of CASI for interviews should have minimized this problem [22, 59]. Finally, this kind of cross-sectional study does not allow us to evaluate the causality of the reported associations. Despite these limitations, our findings have important implications for understanding the risk factors of MA initiation, and the results will help in the development of new prevention or intervention programs for teenagers, adolescents, and adults in Myanmar.

\section{Conclusions}

In conclusion, the current study provides an extensive description of gender similarities and differences in the age of initiation of MA use and factors affecting early initiation in Myanmar. The findings revealed that more than $60.0 \%$ of male and female users started using MA before the age of 18 years. Gender similarities and differences were also found across ethnic groups and with regard to reasons for first-time use of MA, places of first-time use, and risky sexual behaviors. Comprehensive and targeted MA prevention strategies and programs reflecting gender considerations are urgently needed in Myanmar. Moreover, gender-specific health education programs on MA misuse should ideally be implemented before the age of 14 years, which is the age when young people start to increasingly use MA. These programs should especially target ethnic minorities, employed MA users, and vulnerable populations such as bisexual/homosexual men, and those who exchange sex for money and/or drugs. Such programs should also consider entertainment venues as priority areas. Finally, such programs should also promote awareness and knowledge regarding MA and the effects thereof.

\section{Abbreviations \\ AOR: Adjusted odds ratio; ATS: Amphetamine-type stimulants; CASI: Computer assisted self-interviewing; Cl: Confidence intervals; MA: Methamphetamine; RDS: Respondent driven sampling; SD: Standard deviation; STI: Sexually transmitted infection}

\section{Acknowledgements}

The authors would like to thank all the MA users who participated in this study without any hesitation. We also would like to thank Dr. Nyein Chan Aung for his support during data collection.

\section{Funding}

None.

Availability of data and materials

The datasets analyzed during the current study are available from the corresponding author on reasonable request.

\section{Authors' contributions}

YMS TNS, JY, and MJ designed the study. YMS, TNS, NC, NPEK, WK, and SMC conducted the field work. YM, TNS, NC, and JY performed the analysis and interpreted the results. YMS, TNS, NPEK, NC, WT, JY, SMC, and MJ contributed to the writing of the manuscript and approved the final manuscript.

\section{Competing interests}

The authors declare that they have no competing interests.

Consent for publication

Not applicable.

\section{Ethics approval and consent to participate}

The study protocol and consent procedure were approved by the Research Ethics Committee of the Graduate School of Medicine, the University of Tokyo, Tokyo, Japan (Ref. no: 10006/2012), and the Ethical Review Committee, the Defence Services Medical Research Centre, Nay Pyi Taw, Myanmar (Document No: 1/2//RB-7/2013). The purpose of the study and CASI interview procedures were clearly explained to each participant prior to the interview by research team members, and informed consent was taken from all participants.

\section{Publisher's Note}

Springer Nature remains neutral with regard to jurisdictional claims in published maps and institutional affiliations.

\section{Author details}

${ }^{1}$ Department of Healthcare Administration, Graduate School of Medicine, Nagoya University, 65 Tsurumai-cho, Showa-ku, Nagoya 466-8550, Japan. ${ }^{2}$ Nagoya University Asian Satellite Campuses Institute, Nagoya, Japan. ${ }^{3}$ Myanma Perfect Research, Yangon, Myanmar. ${ }^{4}$ Department of Community and Global Health, Graduate School of Medicine, University of Tokyo, Tokyo, Japan. ${ }^{5}$ Department of Social Research, Defence Services Medical Research Centre, Tatkone Township, Nay Pyi Taw, Myanmar. ${ }^{6}$ Institute for Drug and Alcohol Studies, Virginia Commonwealth University, Richmond, VA, USA.

${ }^{7}$ Save the Children, Bahan Township, Yangon, Myanmar.

Received: 19 December 2016 Accepted: 26 April 2017

Published online: 08 May 2017

\section{References}

1. UNODC: Amphetamines and Ecstasy: 2011 Global ATS assessment. Vienna: United Nations Office on Drugs and Crime; 2011.

2. Cretzmeyer M, Sarrazin MV, Huber DL, Block RI, Hall JA. Treatment of methamphetamine abuse: research findings and clinical directions. J Subst Abuse Treat. 2003;24(3):267-77. 
3. Barr AM, Panenka WJ, MacEwan GW, Thornton AE, Lang DJ, Honer WG, Lecomte T. The need for speed: an update on methamphetamine addiction. J Psychiatry Neurosci. 2006;31(5):301-13.

4. McKetin R, Kozel N, Douglas J, Ali R, Vicknasingam B, Lund J, Li JH. The rise of methamphetamine in Southeast and East Asia. Drug Alcohol Rev. 2008; 27(3):220-8.

5. UNODC: World Drug Report 2015. Vienna: United nations Office on Drugs and Crime; 2015.

6. Saw YM, Poudel KC, Kham NP, Chan N, Cope JE, Wai KM, Tun S, Saw TN. Assessment of HIV testing among young methamphetamine users in Muse, Northern Shan State, Myanmar. BMC Public Health. 2014;14(1):735.

7. Rawson RA, Washton A, Domier CP, Reiber C. Drugs and sexual effects: role of drug type and gender. J Subst Abuse Treat. 2002;22(2):103-8.

8. Strauss SM, Falkin GP. Women offenders who use and deal methamphetamine: implications for mandated drug treatment. Women Crim Just. 2001;12(4):77-97

9. Brecht ML, O'Brien A, von Mayrhauser C, Anglin MD. Methamphetamine use behaviors and gender differences. Addict Behav. 2004;29(1):89-106.

10. Kim JY, Fendrich M. Gender differences in juvenile arrestees' drug use, selfreported dependence, and perceived need for treatment. Psychiatr Serv. 2002:53(1):70-5.

11. Dluzen DE, Liu B. Gender differences in methamphetamine use and responses: a review. Gend Med. 2008:5(1):24-35.

12. Helschober B, Miller MA. Methamphetamine abuse in California. NIDA Res Monogr. 1991;115:60-71.

13. Becker JB, Hu M. Sex differences in drug abuse. Front Neuroendocrinol. 2008;29(1):36-47.

14. Becker JB, Perry AN, Westenbroek C. Sex differences in the neural mechanisms mediating addiction: a new synthesis and hypothesis. Biol Sex Differ. 2012;3(1):14.

15. Ellickson PL, Tucker JS, Klein DJ. Ten-year prospective study of public health problems associated with early drinking. Pediatrics. 2003;111(5 Pt 1):949-55.

16. McGue M, lacono WG, Legrand LN, Malone S, Elkins I. Origins and consequences of age at first drink. I. Associations with substance-use disorders, disinhibitory behavior and psychopathology, and P3 amplitude. Alcohol Clin Exp Res. 2001;25(8):1156-65.

17. Brook JS, Balka EB, Whiteman M. The risks for late adolescence of early adolescent marijuana use. Am J Public Health. 1999;89(10):1549-54.

18. Silins E, Hutchinson D, Swift W, Slade T, Toson B, Rodgers B. Factors associated with variability and stability of cannabis use in young adulthood. Drug Alcohol Depend. 2013;133(2):452-8.

19. UNODC: Myanmar Situation Assessment on Amphetamine-Type Stimulants (ATS) 2010. Global SMART Program. Vienna: United nations Office on Drugs and Crime; 2010

20. Saw YM, Yasuoka J, Saw TN, Poudel KC, Tun S, Jimba M. What are the factors associated with HIV testing among male injecting and non-injecting drug users in Lashio, Myanmar: a cross-sectional study. BMJ Open. 2013; doi: 10.1136/bmjopen-2013-002747.

21. Ghanem KG, Hutton HE, Zenilman JM, Zimba R, Erbelding EJ. Audio computer assisted self interview and face to face interview modes in assessing response bias among STD clinic patients. Sex Transm Infect. 2005;81(5):421-5.

22. Metzger DS, Koblin B, Turner C, Navaline H, Valenti F, Holte S, Gross M, Sheon A, Miller H, Cooley P, et al. Randomized controlled trial of audio computer-assisted self-interviewing: utility and acceptability in longitudinal studies. HIVNET Vaccine Preparedness Study Protocol Team. Am J Epidemiol. 2000;152(2):99-106.

23. Bangsberg DR, Bronstone A, Chesney MA, Hecht FM. Computer-assisted self-interviewing (CASI) to improve provider assessment of adherence in routine clinical practice. J Acquir Immune Defic Syndr. 2002;31 Suppl 3: S107-111.

24. Paz-Bailey G, Jacobson JO, Guardado ME, Hernandez FM, Nieto Al, Estrada M, Creswell J. How many men who have sex with men and female sex workers live in El Salvador? Using respondent-driven sampling and capturerecapture to estimate population sizes. Sex Transm Infect. 2011;87(4):279-82.

25. UNODC: Amphetamine-type stimulants in Viet Nam: a review of the availability, use and implications for health and security in Viet Nam. Viet Nam: United Nations Office on Drugs and Crime, Viet Nam Country Office; 2012.

26. Kecojevic A, Wong CF, Schrager SM, Silva K, Bloom JJ, Iverson E, Lankenau SE. Initiation into prescription drug misuse: differences between lesbian, gay, bisexual, transgender (LGBT) and heterosexual high-risk young adults in Los Angeles and New York. Addict Behav. 2012;37(11):1289-93.
27. UNESCO-IBE: World data on education 7th ed. 2010/2011. Geneva: United Nations Educational, Scientific and Cultural Organizations; International Bureau of Education; 2011.

28. Gibbons FX, Gerrard M, Vande Lune LS, Wills TA, Brody G, Conger RD. Context and cognitions: environmental risk, social influence, and adolescent substance use. Pers Soc Psychol Bull. 2004;30(8):1048-61.

29. Gil AG, Vega WA, Biafora F. Temporal influences of family structure and family risk factors on drug use initiation in a multiethnic sample of adolescent boys. J Youth Adolesc. 1998;27:373-93.

30. Brecht ML, Greenwell L, Anglin MD. Substance use pathways to methamphetamine use among treated users. Addict Behav. 2007;32(1): 24-38.

31. Gillmore MR, Catalano RF, Morrison DM, Wells EA, Iritani B, Hawkins JD. Racial differences in acceptability and availability of drugs and early initiation of substance use. Am J Drug Alcohol Abuse. 1990;16(3-4):185-206.

32. Ellickson PL, Morton SC. Identifying adolescents at risk for hard drug use: racial/ethnic variations. J Adolesc Health. 1999;25(6):382-95.

33. Duncan AE, Lessov-Schlaggar CN, Sartor CE, Bucholz KK. Differences in time to onset of smoking and nicotine dependence by race/ethnicity in a Midwestern sample of adolescents and young adults from a high risk family study. Drug Alcohol Depend. 2012:125(1-2):140-5.

34. Saw YM, Saw TN, Wai KM, Poudel KC, Win HH. Correlates of sex trading among male non-injecting drug users in Myanmar: a cross-sectional study. Harm Reduct J. 2016;13(1):34.

35. Zaluar A. Violence in Rio de Janeiro: styles of leisure, drug use, and trafficking. Int Soc Sci J. 2001;53(3):369-78.

36. Kelly BC, Leclair A, Parsons JT. Methamphetamine use in club subcultures. Subst use misuse. 2013;48(14):1541-52.

37. Mansergh G, Colfax GN, Marks G, Rader M, Guzman R, Buchbinder S. The Circuit Party Men's Health Survey: findings and implications for gay and bisexual men. Am J Public Health. 2001;91(6):953-8.

38. Colfax GN, Mansergh G, Guzman R, Vittinghoff E, Marks G, Rader M Buchbinder S. Drug use and sexual risk behavior among gay and bisexual men who attend circuit parties: a venue-based comparison. J Acquir Immune Defic Syndr. 2001;28(4):373-9.

39. Kokkevi A, Loukadakis M, Plagianakou S, Politikou K, Stefanis C. Sharp increase in illicit drug use in Greece: trends from a general population survey on licit and illicit drug use. Eur Addict Res. 2000;6(1):42-9.

40. Van Havere T, Vanderplasschen W, Lammertyn J, Broekaert E, Bellis M. Drug use and nightlife: more than just dance music. Subst Abuse Treat Prev Policy. 2011;6:18

41. Boeri MW, Sterk CE, Elifson KW. Rolling beyond raves: ecstasy use outside the rave setting. J Drug Issues. 2004;34(4):831-60.

42. Wu LT, Pilowsky DJ, Schlenger WE, Galvin DM. Misuse of methamphetamine and prescription stimulants among youths and young adults in the community. Drug Alcohol Depend. 2007;89(2-3):195-205.

43. Westermeyer J, Boedicker AE. Course, severity, and treatment of substance abuse among women versus men. Am J Drug Alcohol Abuse. 2000;26(4):523-35.

44. Lin SK, Ball D, Hsiao CC, Chiang YL, Ree SC, Chen CK. Psychiatric comorbidity and gender differences of persons incarcerated for methamphetamine abuse in Taiwan. Psychiatry Clin Neurosci. 2004; 58(2):206-12.

45. Hser Yl, Evans E, Huang YC. Treatment outcomes among women and men methamphetamine abusers in California. J Subst Abuse Treat. 2005;28(1):77-85

46. UNODC. Drugs and HIV/AIDS Country Programme (2009-2010). Myanmar: United Nations Office on Drugs and Crime; 2008.

47. UNODC. Substance abuse treatment and care for women: Case studies and lessons learned. Vienna, Austria: United Nations Office on Drugs and Crime; 2004

48. Hecksher D, Hesse M. Women and substance use disorders. Mens Sana Monogr. 2009;7(1):50-62.

49. Van Etten ML, Anthony JC. Male-female differences in transitions from first drug opportunity to first use: searching for subgroup variation by age, race, region, and urban status. J Womens Health Gend Based Med. 2001;10(8):797-804.

50. Parsons JT, Kelly BC, Weiser JD. Initiation into methamphetamine use for young gay and bisexual men. Drug Alcohol Depend. 2007;90(2-3):135-44.

51. Semple SJ, Patterson TL, Grant I. The context of sexual risk behavior among heterosexual methamphetamine users. Addict Behav. 2004;29(4):807-10. 
52. Machado EC, Silveira MF, Silveira VM. Prevalence of weight-loss strategies and use of substances for weight-loss among adults: a population study. Cad Saude Publica. 2012;28(8):1439-49.

53. Cookson J, Silverstone T. The effects of methylamphetamine on mood and appetite in depressed patients: a placebo-controlled study. Int Clin Psychopharmacol. 1986;1(2):127-33.

54. Pisetsky EM, Chao YM, Dierker LC, May AM, Striegel-Moore RH. Disordered eating and substance use in high-school students: results from the Youth Risk Behavior Surveillance System. Int J Eat Disord. 2008;41(5):464-70.

55. Piper B. A four-pillars approach to Methamphetamine: policies for effective drug prevention, treatment, policing and harm reduction. the United States of America: The Drug Policy Alliance; 2008.

56. Weber AE, Boivin JF, Blais L, Haley N, Roy E. HIV risk profile and prostitution among female street youths. J Urban Health. 2002;79(4):525-35.

57. Logan TK, Leukefeld C, Farabee D. Sexual and drug use behaviors among women crack users: implications for prevention. AIDS Educ Prev. 1998; 10(4):327-40.

58. Tourangeau R, Rips LJ, Rasinski K. The Psychology of urvey Response. New York: Cambridge University Press; 2000.

59. Turner CF, Ku L, Rogers SM, Lindberg LD, Pleck JH, Sonenstein FL. Adolescent sexual behavior, drug use, and violence: increased reporting with computer survey technology. Science. 1998;280(5365):867-73.

Submit your next manuscript to BioMed Central and we will help you at every step:

- We accept pre-submission inquiries

- Our selector tool helps you to find the most relevant journal

- We provide round the clock customer support

- Convenient online submission

- Thorough peer review

- Inclusion in PubMed and all major indexing services

- Maximum visibility for your research

Submit your manuscript at www.biomedcentral.com/submit

\section{(O) BioMed Central}

